\title{
Sources of Klebsiella and Raoultella species on dairy farms: Be careful where you walk
}

\author{
R. N. Zadoks, ${ }^{* 1,2}$ H. M. Griffiths, ${ }^{* 3}$ M. A. Munoz, ${ }^{* 4}$ C. Ahlstrom, ${ }^{* 5}$ G. J. Bennett, ${ }^{\star}$ E. Thomas, $\dagger^{6}$ \\ and Y. H. Schukken* \\ ${ }^{*}$ Quality Milk Production Services, College of Veterinary Medicine, Cornell University, Ithaca, NY 14850 \\ tW. H. Miner Agricultural Research Institute, Chazy, NY 12921
}

\begin{abstract}
Klebsiella spp. are a common cause of mastitis, milk loss, and culling on dairy farms. Control of Klebsiella mastitis is largely based on prevention of exposure of the udder to the pathogen. To identify critical control points for mastitis prevention, potential Klebsiella sources and transmission cycles in the farm environment were investigated, including oro-fecal transmission, transmission via the indoor environment, and transmission via the outdoor environment. A total of 305 samples was collected from 3 dairy farms in upstate New York in the summer of 2007, and included soil, feed crops, feed, water, rumen content, feces, bedding, and manure from alleyways and holding pens. Klebsiella spp. were detected in $100 \%$ of rumen samples, $89 \%$ of water samples, and approximately $64 \%$ of soil, feces, bedding, alleyway, and holding pen samples. Detection of Klebsiella spp. in feed crops and feed was less common. Genotypic identification of species using rpoB sequence data showed that Klebsiella pneumoniae was the most common species in rumen content, feces, and alleyways, whereas Klebsiella oxytoca, Klebsiella variicola, and Raoultella planticola were the most frequent species among isolates from soil and feed crops. Random amplified polymorphic DNA-based strain typing showed heterogeneity of Klebsiella spp. in rumen content and feces, with a median of 4 strains per 5 isolates. Observational and bacteriological data support the existence of an oro-fecal transmission cycle, which is primarily
\end{abstract}

Received July 7, 2010.

Accepted October 14, 2010.

${ }^{1}$ Corresponding author: ruth.zadoks@moredun.ac.uk

${ }^{2}$ Current address: Moredun Research Institute, Penicuik, UK; and Royal (Dick) School of Veterinary Studies, University of Edinburgh, Roslin, UK.

${ }^{3}$ Current address: Department of Plant Pathology and PlantMicrobe Biology, Cornell University, Ithaca, NY 14850.

${ }^{4}$ Current address: Facultad de Ciencias Veterinarias, Universidad de Concepción, Chillán, Chile.

${ }^{5}$ Current address: Department of Animal Sciences, Colorado State University, Fort Collins, CO 80523.

${ }^{6}$ Current address: 82 Dake Circle, Hammond, NY 13646. maintained through direct contact with fecal contamination or through ingestion of contaminated drinking water. Fecal shedding of Klebsiella spp. contributes to pathogen loads in the environment, including bedding, alleyways, and holding pens. Hygiene of alleyways and holding pens is an important component of Klebsiella control on dairy farms.

Key words: Klebsiella, environment, bedding, hygiene

\section{INTRODUCTION}

Mastitis caused by gram-negative pathogens is a major concern on many well-managed dairy farms in North America. The main gram-negative pathogens that cause mastitis are Escherichia coli and Klebsiella spp. (Wenz et al., 2001; Roberson et al., 2004; Olde Riekerink et al., 2008). Cows with Klebsiella spp. mastitis are more likely to die or to be culled than are cows with other types of mastitis (Erskine et al., 2002; Gröhn et al., 2005; Wilson et al., 2007). Klebsiella spp. mastitis causes a considerable and often sustained decrease in milk production, with average losses of 7.6 $\mathrm{kg} / \mathrm{d}$ shortly after infection and $5 \mathrm{~kg} / \mathrm{d}$ in subsequent months (Gröhn et al., 2004). Klebsiella spp. mastitis responds poorly to antimicrobial treatment (Roberson et al., 2004). Vaccination has been reported to provide some protection against the risk of culling but does not affect the incidence or severity of clinical mastitis due to Klebsiella spp. (Wilson et al., 2007). Thus, prevention of exposure is the main strategy for control of Klebsiella spp. mastitis.

Sawdust and shavings are important sources of Klebsiella spp. (Newman and Kowalski, 1973; National Mastitis Council, 1999). Growth of Klebsiella spp. is also observed on mattresses without shavings or other bedding (Kristula et al., 2008). In recent years, Klebsiella spp. mastitis has become a problem in herds that use sand bedding. This is attributed to fecal shedding of Klebsiella spp. by healthy adult dairy cattle (Munoz et al., 2006). Fecal shedding of Klebsiella spp. does not appear to be associated with a chronic carrier state 
Table 1. Characteristics of dairy farms participating in Klebsiella spp. study in Northern New York

\begin{tabular}{|c|c|c|c|}
\hline Item & \multicolumn{3}{|c|}{ Farm } \\
\hline County & St. Lawrence & Franklin & Clinton \\
\hline Production $(\mathrm{kg} / \mathrm{d})$ & 35.0 & 37.3 & 38.6 \\
\hline Bulk milk SCC (cells/mL) & 363,500 & 377,500 & 290,000 \\
\hline \multicolumn{4}{|l|}{ Bedding } \\
\hline Feeding system & TMR in drive-through bunks & TMR in drive-through bunks & TMR in drive-through bunks \\
\hline Manure handling & Alley scrapers, set to scrape $2 \times$ per $\mathrm{h}$ & $\begin{array}{l}\text { Alley scrapers set to scrape } 1 \times \text { per } \\
\mathrm{h} \text { for some pens; skid steer during } \\
\text { milking }(3 \times) \text { for other pens }\end{array}$ & Alley scrapers, set to scrape $2 \times$ per $\mathrm{h}$ \\
\hline
\end{tabular}

but rather with frequent uptake and high turnover of a large variety of Klebsiella spp. strains that pass through the gastrointestinal tract (Munoz and Zadoks, 2007). In wheat, corn, and alfalfa, Klebsiella pneumoniae can act as an endophyte that helps plants to bind nitrogen and bacteria can be found inside the stems and leaves of plants in the absence of external fecal contamination (Chelius and Triplett, 2000; Dong et al., 2003; Iniguez et al., 2004). Oral intake of Klebsiella spp. could, therefore, be due to the presence of the organism in or on crops used for feed, or to fecal contamination of feed and water (Figure 1). Fecal shedding of Klebsiella spp. resulting in contamination of dairy cattle and their environment is common and it seems likely that exposure of teat ends to Klebsiella spp. could result from contact with sources other than bedding (e.g., contaminated alleyways). If such sources could be identified, this might

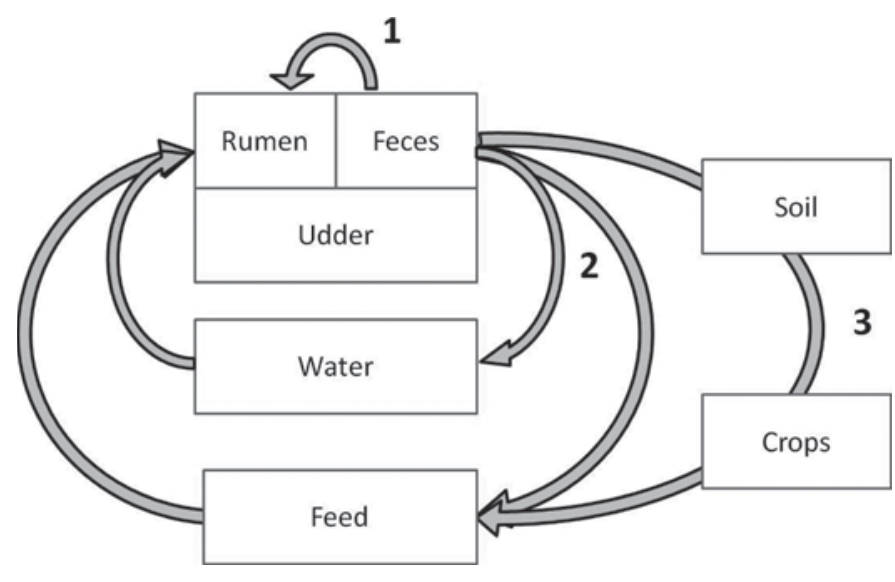

Figure 1. Hypothetical oro-fecal Klebsiella spp. transmission on dairy farms showing (1) direct oro-fecal transmission (e.g., through licking of contaminated body parts); (2) indirect oro-fecal transmission via contaminated water or feed without involvement of the external environment; and (3) transmission via contaminated feed with involvement of the environment (soil, feed, and crops). provide dairy herd managers with additional control points for prevention of Klebsiella spp. mastitis.

In our experience as extension veterinarians, most farmers are aware of the role of bedding as a potential source of Klebsiella spp. but not of the role of other environmental sources. Scientific studies on the presence of Klebsiella spp. in environmental sources other than bedding on dairy farms are rare. The aim of this study was to provide farmers with information on potential Klebsiella spp. transmission cycles in the dairy farm ecosystem, considering oro-fecal transmission, transmission via the indoor farm environment, and transmission via the outdoor farm environment.

\section{MATERIALS AND METHODS}

\section{Farms}

In June, July, August, and September 2007, samples were collected from 3 dairy farms during 1 visit per farm per month. Farms were located in 3 counties in Northern New York. Farm characteristics are summarized in Table 1. Potential for transmission of Klebsiella spp. via an oro-fecal cycle was evaluated by sampling of feces, feed, and water. Potential for transmission of Klebsiella spp. via the indoor environment was evaluated through sampling of bedding, alleyways, and holding pens. For preliminary assessment of the potential for transmission of Klebsiella spp. via the outdoor environment, soil and crop samples were collected.

\section{Sample Collection}

Fecal samples $(\mathrm{n}=71)$ were collected from the rectum using clean plastic sleeves. Rumen content samples (n $=19$ ) were collected from healthy lactating cows with permanently fitted rumen fistulas using clean plastic sleeves. Feed samples included TMR $(\mathrm{n}=12)$ from feeding alleys collected after animals had eaten from it 
and TMR or other feed components $(\mathrm{n}=6)$ sampled before mixing or feeding. Feed samples were collected in clean zip lock bags of appropriate size. Water samples $(\mathrm{n}=15)$ were collected in sterile $240-\mathrm{mL}(8 \mathrm{oz})$ snap cap vials (Capitol Vial, Fultonville, NY). Bedding samples $(\mathrm{n}=57)$ were collected from storage piles and from the back 1/3 of stalls (Munoz et al., 2006). Samples from alleyways and holding pens $(\mathrm{n}=53)$ consisted of manure slurry picked up from floors using plastic bags or snap cap vials, depending on consistency of the slurry. Soil samples $(\mathrm{n}=42)$ were collected from the plough layer of corn and alfalfa plots. Samples from preharvest crops $(\mathrm{n}=19)$ were obtained from the same corn and alfalfa plots as were soil samples. In September, only postharvest crops were available and samples $(\mathrm{n}=10)$ were collected from corn cut from 10 different fields. Soil and plant samples were stored in clean plastic bags of appropriate size. All samples were stored in cooler boxes with icepacks and transported to the laboratory within $30 \mathrm{~h}$ of collection. On-farm activities were conducted with approval from Cornell University's Institutional Animal Care and Use Committee.

\section{Microbiological Analysis}

The presence of Klebsiella spp. in feces, feed, rumen content, samples from alleyways and holding pens, and soil and postharvest feed crops (corn) was detected using the procedures described by Munoz and coworkers (2006). Briefly, a 1:10 dilution of the sample in saline ( $1 \mathrm{~g}$ of sample in $9 \mathrm{~mL}$ of saline) was prepared and incubated for $4 \mathrm{~h}$ at $37^{\circ} \mathrm{C}$ before being streaked onto MacConkey agar containing $10 \mathrm{mg} / \mathrm{L}$ of ampicillin (MacA). Preharvest feed crops (i.e., corn and alfalfa plants) were separated into roots, stems, and leaves. Plant parts were surface sterilized for $10 \mathrm{~s}$ using $2 \%$ of sodium hypochlorite (Clorox Regular-Bleach, The Clorox Company, Oakland, CA) to allow for detection of endophytic Klebsiella spp. After bleaching, plant parts were rinsed twice in sterile distilled water before being ground, diluted 1:10 in saline $(1 \mathrm{~g}$ of sample in $9 \mathrm{~mL}$ of saline), and plated onto MacA. For water samples, $150 \mathrm{~mL}$ was filtered through $20-\mu \mathrm{m}$ Nalgene bottle top filters using a vacuum pump. Filters were taken from bottle tops with sterile tweezers and transferred to a 14-mL culture tube (VWR, West Chester, PA) with 8 $\mathrm{mL}$ of Todd-Hewitt broth (CM0189; Oxoid Ltd., Basingstoke, UK). After incubation for $4 \mathrm{~h}$ at $37^{\circ} \mathrm{C}$, broth was swabbed onto MacA. For bedding samples, $5 \mathrm{~g}$ of bedding was mixed with $45 \mathrm{~mL}$ of saline using a stomacher. Additional 10-fold dilutions were made in saline and dilutions of $10^{-1}, 10^{-2}$, and $10^{-3}$ were streaked onto MacA. Dilutions of bedding samples were not prepared to determine the bacterial load in bedding but to ob- tain a bacterial dilution that would allow for selection of isolated colonies from agar for further processing. For all sample types, saline plated onto MacA was used as negative control, and an arbitrarily selected sample inoculated with $K$. pneumoniae ATCC 13883 was used as a positive control for culture procedures. For all sample types, inoculated MacA plates were incubated for 24 to $48 \mathrm{~h}$ at $37^{\circ} \mathrm{C}$. Putative Klebsiella spp. colonies were identified based on colony morphology (Munoz et al., 2006). Based on citrate, motility, and indole testing, isolates were classified as K. pneumoniae (citrate positive, motility negative, indole negative) or Klebsiella oxytoca (citrate positive, motility negative, indole positive; National Mastitis Council, 1999). A sample was considered positive if a single colony of Klebsiella spp. was detected (Munoz et al., 2006).

\section{Molecular Analysis}

Previous on-farm studies demonstrated presence of multiple Klebsiella and Raoultella spp. in bedding, feces, and water but samples from rumen content, soil, and plants were not tested in those studies (Munoz et al., 2006, 2007). Phenotypic methods do not differentiate Klebsiella variicola or Raoultella spp. from K. pneumoniae and $K$. oxytoca. In the current study, genotypic methods (i.e., rpoB sequencing; Munoz et al., 2007) were, therefore, used for species identification of isolates from rumen $(\mathrm{n}=73)$, soil $(\mathrm{n}=37)$, and plants $(\mathrm{n}$ $=27$ ). In addition to rumen isolates, fecal isolates $(\mathrm{n}=$ 51) from the same animals were genotypically identified at species level as well as a limited number of alleyway isolates $(\mathrm{n}=15)$ from the same farm. Random amplified polymorphic DNA (RAPD) typing (Munoz and Zadoks, 2007) was used to assess and compare strain heterogeneity within paired rumen and fecal samples (i.e., samples obtained from the same cow on the same day). Per sample, 5 isolates were used and RAPD-PCR products from paired rumen and fecal samples were run side by side on agarose gels to allow for direct comparison of banding patterns. Patterns were read by 2 independent observers and any discrepancies in interpretation were resolved as described previously (Munoz et al., 2007).

\section{RESULTS}

\section{Feces and Rumen Samples}

Of 52 feces samples collected in June, $67 \%$ tested positive for Klebsiella spp. (Figure 2). Positive samples were detected on each farm. Paired feces and rumen content samples were collected from 12 animals in August and September. Seven animals were sampled twice 


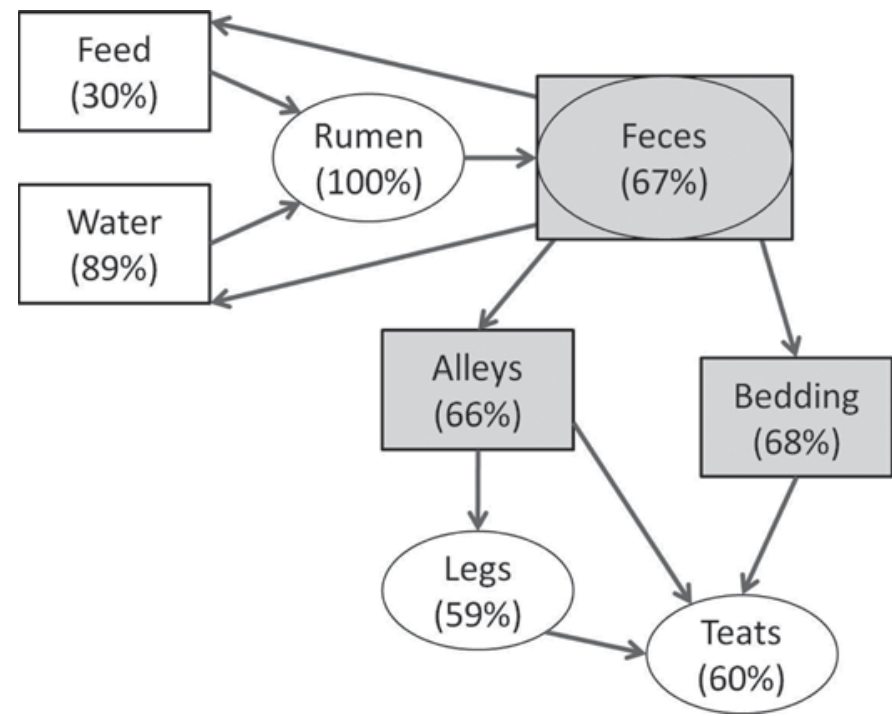

Figure 2. Potential sources and routes of exposure of the mammary gland to Klebsiella spp. Ovals represent animals (rectum/feces, rumen, legs, and teats). Rectangles represent environmental sources. Gray rectangles are considered of primary importance for farm management and mastitis prevention. Proportion of samples testing positive based on 3 New York dairy farms described in the current study (feed, water, rumen, feces, alleys, bedding) and Munoz et al. (2008; legs, teats)

and 5 animals were sampled once, yielding a total of 19 sample pairs. All rumen content samples (100\%) and 16 of 19 fecal samples (84\%) tested positive for Klebsiella spp. Based on rpoB sequencing, K. pneumoniae was the dominant species in both sample types. In addition, $K$. variicola was detected in both sample types. Klebsiella oxytoca and Raoultella planticola were only detected in rumen content (Table 2). In phenotypic tests, $K$. variicola was identified as K. pneumoniae, whereas $R$. planticola could have the appearance of $K$. pneumoniae (indole negative) or K. oxytoca (indole positive). For 10 cows, 5 rumen isolates and 5 fecal isolates were available from paired samples. Using RAPD typing, between 3 and 5 strains (median $=4$ ) were detected per rumen sample and between 1 and 5 strains $($ median $=4$ ) were detected per fecal sample. Within 4 of 10 animals, shar-

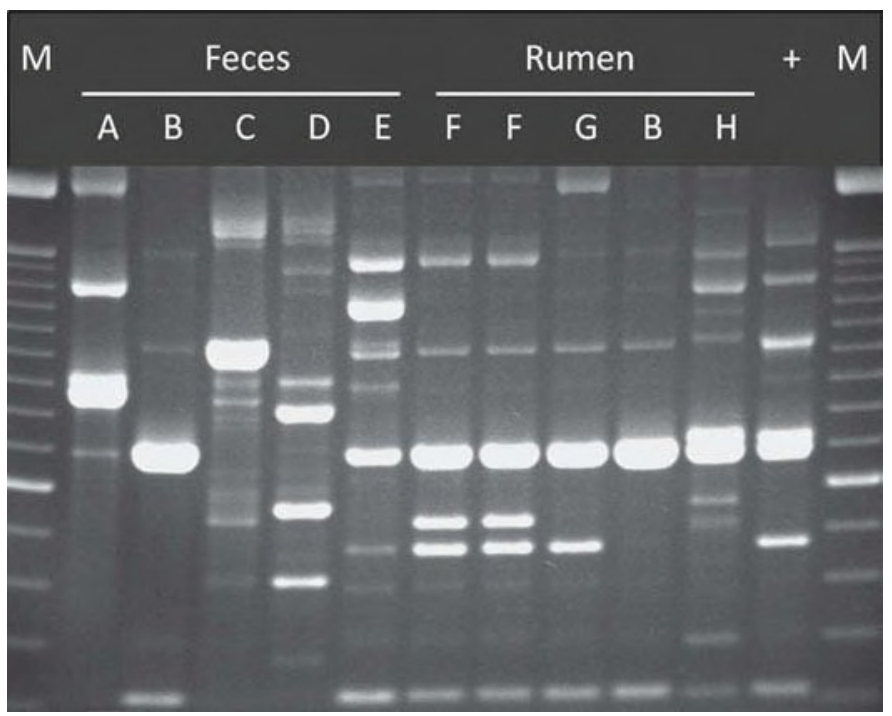

Figure 3. Comparison of Klebsiella spp. isolates from feces and rumen content from a single cow on a single day. $\mathrm{M}=$ molecular size marker; + = positive control (ATCC 13883); A through $\mathrm{H}=$ random amplified polymorphic DNA patterns.

ing of an RAPD pattern between paired rumen and fecal samples was observed (Figure 3).

\section{Feed and Water Samples}

Eight of nine water samples (89\%; Figure 2) from drinking troughs were positive for Klebsiella spp. Positive samples were detected on each farm. Source water used to fill the troughs was tested on one farm, and Klebsiella spp. were not detected. Four of twelve TMR samples collected from feeding alleys (30\%; Figure 2$)$ and hay $(\mathrm{n}=1)$ and straw $(\mathrm{n}=1)$ collected from storage piles were positive for Klebsiella spp. Positive TMR samples were detected on each farm. Haylage $(\mathrm{n}=1)$ and corn silage $(\mathrm{n}=3)$ samples originating from storage piles on a single farm tested negative for Klebsiella spp.

\section{Bedding and Alley Samples}

Of 53 samples of manure or slurry collected from floors in barns, holding pens, and parlors, $66 \%$ tested

Table 2. Distribution of Klebsiella and Raoultella species (number of isolates; percentage within sample type in parentheses) identified by rpoB sequencing among samples from animals, the internal environment, and the external environment of farm $\mathrm{C}$

\begin{tabular}{|c|c|c|c|c|c|c|}
\hline Source & Samples & Isolates & $\begin{array}{c}\text { Klebsiella } \\
\text { oxytoca }\end{array}$ & $\begin{array}{c}\text { Klebsiella } \\
\text { pneumoniae }\end{array}$ & $\begin{array}{c}\text { Klebsiella } \\
\text { variicola }\end{array}$ & $\begin{array}{l}\text { Raoultella } \\
\text { planticola }\end{array}$ \\
\hline Soil & 19 & 37 & $13(35)$ & $4(11)$ & $2(5)$ & $18(49)$ \\
\hline Plants $^{1}$ & 10 & 27 & $2(7)$ & $4(15)$ & $18(67)$ & 3 (11) \\
\hline Rumen & 19 & 73 & $1(1)$ & $67(92)$ & $3(4)$ & $2(3)$ \\
\hline Feces & 16 & 51 & $0(0)$ & $48(94)$ & $3(6)$ & $0(0)$ \\
\hline Alleyways & 13 & 15 & $0(0)$ & $15(100)$ & $0(0)$ & $0(0)$ \\
\hline
\end{tabular}

${ }^{1}$ Includes 2 isolates from alfalfa roots (preharvest) and 25 isolates from freshly cut corn originating from 8 corn fields (postharvest). 
positive for Klebsiella spp. (Figure 2). Positive samples were detected on all farms and originated from alleys within and between pens and parlors, holding pens, and a footbath. Of 57 bedding samples, $68 \%$ tested positive for Klebsiella spp. (Figure 2). This count includes 5 unused bedding samples of which 2 tested positive for Klebsiella spp.

\section{Soil and Plant Samples}

Members of the genus Klebsiella or Raoultella were found in 26 of 42 soil samples. Soil samples originated from the same farm and from a corn field that received annual manure applications ( $\mathrm{n}=18$ samples, $67 \%$ positive), an alfalfa field that received annual manure applications ( $\mathrm{n}=21$ samples, $57 \%$ positive), and an alfalfa field that had not received manure applications for 50 years ( $\mathrm{n}=3$ samples of which 2 were positive). Per soil sample, multiple isolates were identified to species level using rpoB sequencing. Raoultella planticola was the most common species in soil samples, followed by $K$. oxytoca (Table 2). Surface sterilized leaves or stems from corn and alfalfa collected preharvest $(\mathrm{n}=$ 10 and 9 plants, respectively) did not yield Klebsiella or Raoultella spp. Among surface sterilized roots from the same plants, 2 alfalfa samples tested positive for $K$. oxytoca. Most postharvest corn samples (8 of 10) showed heavy growth of Klebsiella spp., with K. variicola as the dominant species (Table 2).

\section{DISCUSSION}

This study was designed and conducted in consultation with dairy farmers to address their questions about sources of Klebsiella spp. on dairy farms that may contribute to exposure of the mammary gland and to ingestion and fecal excretion of the organisms. Results from this study show that Klebsiella spp. are commonly present in environmental sources other than bedding, most importantly in alleyways and holding pens (Figure 2). Presence of Klebsiella spp. throughout the dairy farm environment could be expected based on its frequent presence in feces (Munoz et al., 2006, 2007), but participating farmers were not aware of this. We observed that even on farms where managers paid close attention to bedding hygiene and bedding replacement frequency, alley hygiene and maintenance of alley scrapers were neglected. Fecal contamination of the udder, which may be caused by cow traffic via dirty alleyways and holding pens, is associated with increased risk of presence of Klebsiella spp. on teat skin before and during milking (Munoz et al., 2008). Anecdotal evidence from farm B collected during the study suggested that improved alley hygiene resulted in lower levels of teat contamination and lower levels of Klebsiella spp. detection after premilking udder preparation. In the current study, levels of Klebsiella spp. contamination were quantified as prevalence within sample type. Further quantification of bacterial loads (e.g., cfu counts in samples from different environmental sources), could refine our understanding of the relative importance of bedding, alleys, or other sources in the transmission of Klebsiella spp. The simple message, however, is that Klebsiella spp. are found wherever manure is found and that bedding management is not sufficient to avoid exposure of teats to these potential mastitis pathogens.

With growing awareness that fecal contamination of the environment is an important source of Klebsiella spp., farmers subsequently want to know how Klebsiella spp. get into feces, and how fecal shedding of Klebsiella spp. might be reduced. Passage of Klebsiella spp. from feed to the gut would require survival of the bacteria in the acidic environment of the abomasum, unless the bacteria were protected inside undigested plant matter. Undigested feed materials can reach the gut (Russell et al., 2000) and Klebsiella spp. can be present inside feed crops, where they act as endophytes (Chelius and Triplett, 2000). In the current study, based on testing of a limited number of plant samples, endophytic presence of Klebsiella spp. inside feed crops could only be detected in roots, parts of the plant that would normally not be eaten by cattle. Protection from gastric acid may also be provided by mixed growth of enterobacteriaceae with acid tolerant bacteria and biofilms (O'May et al., 2005). Survival of Klebsiella spp. at low pH may furthermore be enhanced by induction of acid tolerance, a phenomenon that has been described by Aghi and Chhibber (1999). Acid tolerance was induced at $\mathrm{pH} 5.0$ and enhanced bacterial survival at $\mathrm{pH} 3.5$ (Aghi and Chhibber, 1999). One could speculate that low rumen $\mathrm{pH}$, particularly in the case of subclinical rumen acidosis, might, thus, enhance survival of Klebsiella spp. during passage from the rumen to the gut. For E. coli in cattle and for Klebsiella spp. in humans, high starch content in the diet has been claimed to increase fecal shedding of the organism of interest (Russell et al., 2000; Ebringer et al., 2007). Alternatively, or in addition, high rates of passage through the gastrointestinal tract due to high feed volumes may result in decreased killing of bacteria by gastric acid and bile products. Whether increased starch content in bovine rations or increased feed volumes contribute to increased Klebsiella spp. levels in the bovine gastrointestinal tract was not investigated. We did demonstrate for the first time, however, that Klebsiella spp. were present in the rumen of all cows that were tested, and that multiple species and strains were present in a single gram of rumen content. Both species that were predominantly soil or plant 
associated in our study (i.e., K. variicola and R. planticola; Table 2), and the predominant species from fecal and environmental samples (i.e., K. pneumoniae), were present in rumen content. Heterogeneity of Klebsiella populations in the gastrointestinal tract is high (Figure 3; Döpfer et al., 2008) and as many as 15 isolates per sample might need to be typed to identify all strains present in that sample with $95 \%$ certainty. Against that background, the fact that identical RAPD types were identified in rumen and fecal samples of some cows would appear to suggest that there is flow-through of Klebsiella spp. from the rumen to the gut. The flora of farm soil and postharvest crops was dominated by $K$. variicola and $R$. planticola, species that were relatively rare in the rumen. It is unknown whether the rumen does not support the survival or growth of these species to the same extent that it supports survival and growth of $K$. pneumoniae, or whether the rumen is predominantly populated through reingestion of fecal flora, which is also dominated by K. pneumoniae. In our opinion, ingestion of Klebsiella spp. is most likely due to fecal contamination of feed and water, both because of the prevalence and distribution of bacterial species in different sample types, and because of the likelihood of contact with the various sample types. Further studies would be needed to better understand the levels and relevance of Klebsiella species in forage crops and preserved feed and to explore strategies to control levels of ingestion and excretion of Klebsiella spp. Such studies could be epidemiological in nature, focusing on identification of herd- or management-level risk factors associated with fecal shedding, or physiological (e.g., focusing on the effect of rations and rumen acidosis on fecal shedding). In addition, the effect of water disinfection and feed storage conditions and preservation methods could be explored. Whether manipulation of ingestion or fecal shedding of Klebsiella spp. is technically feasible and economically viable remains to be seen. Meanwhile, knowing that the majority of healthy cows shed Klebsiella spp. in feces, farmers should be made aware that bedding is only one of many possible sources of Klebsiella spp. and that manure in alleyways and holding pens can contribute to high Klebsiella spp. levels on teat skin (Munoz et al., 2008). For practical purposes, hygiene of alleyways and holding pens is of far greater importance than manipulation of dietary ingestion or fecal excretion levels.

In the current study, the genera Klebsiella and Raoultella were captured under the name Klebsiella spp. Although scientifically incorrect, it is common practice in mastitis diagnostics to group pathogens that are phenotypically similar under a single genus name, even if they belong to different genera. Another example would be use of the names Streptococcus spp. or environmental streptococci for species of the genera Streptococcus, Enterococcus, and Lactococcus. Raoultella planticola, formerly known as K. planticola (Drancourt et al., 2001), was phenotypically indistinguishable from K. oxytoca and K. pneumniae and would be classified as a Klebsiella in routine diagnostics. Other Raoultella species also look like Klebsiella spp. on phenotypic testing (Granier et al., 2003; Munoz et al., 2007). In the current study, $R$. planticola was most common in soil and plant samples, consistent with its previously described association with plants, soil, and water (Drancourt et al., 2001). Raoultella spp. have also been identified in rumen content (this study) and bedding (Munoz et al., 2007). In humans, Raoultella spp. can be of clinical relevance (Granier et al., 2003). To our knowledge, they have not been associated with clinical mastitis or other diseases in cattle but without use of genotypic identification methods, their prevalence and effect may be underestimated.

\section{CONCLUSIONS}

Klebsiella spp. are commonly present in soil, water troughs, rumen content, feces, bedding, alleyways, and holding pens on dairy farms. Predominant species in soil and plant samples differ from those in the gastrointestinal tract, water, bedding, alleyways, and holding pens, and the presence of Klebsiella spp. in plants appears to result from external contamination rather than endophytic colonization of feed crops. Oro-fecal transmission due to fecal contamination of animals, feed, and water appears more important for maintenance of Klebsiella spp. in the farm ecosystem than does introduction from external sources such as fresh bedding or feed. Fecal contamination of stalls, alleyways, and holding pens may result in exposure of teat ends to Klebsiella. Further studies would be needed to identify methods to reduce oral ingestion and fecal shedding of Klebsiella spp. Meanwhile, hygiene of alleyways and holding pens should be recognized as an important component of prevention of mastitis due to Klebsiella spp.

\section{ACKNOWLEDGMENTS}

This study was funded by the Northern New York Agricultural Development Program. We thank staff at participating farms and Quality Milk Production Services locations and at the W. H. Miner Agricultural Research Institute, Chazy, New York, for their collaboration, questions and support.

\section{REFERENCES}

Aghi, P., and S. Chhibber. 1999. Adaptive acid tolerance in Klebsiella pneumoniae. Indian J. Med. Microbiol. 17:81-84. 
Chelius, M. K., and E. W. Triplett. 2000. Immunolocalizatoin of dinitrogenase reductase produced by Klebsiella pneumoniae in association with Zea mays. L. Appl. Environ. Microbiol. 66:783-787.

Dong, Y., A. L. Iniguez, B. M. Ahmer, and E. W. Triplett. 2003 Kinetics and strain specificity of rhizosphere and endophytic colonization by enteric bacteria on seedlings of Medicago sativum and Medicago truncatula. Appl. Environ. Microbiol. 69:1783-1790.

Döpfer, D., W. Buist, Y. Soyer, M. A. Munoz, R. N. Zadoks, L. Geue, and B. Engel. 2008. Assessing genetic heterogeneity within bacterial species isolated from gastrointestinal and environmental samples: How many isolates does it take? Appl. Environ. Microbiol. 74:3490-3496.

Drancourt, M., C. Bollet, A. Carta, and P. Rousselier. 2001. Phylogenetic analyses of Klebsiella species delineate Klebsiella and Raoultella gen. nov. with description of Raoultella ornitholytica comb. nov., Raoultella terrigena comb. nov. and Raoultella planticola comb. nov. Int. J. Syst. Evol. Microbiol. 51:925-932.

Ebringer, A., T. Rashid, H. Tiwana, and C. A. Wilson. 2007. A possible link between Crohn's disease and ankylosing spondylitis via Klebsiella infections. Clin. Rheumatol. 26:289-297.

Erskine, R. J., P. C. Bartlett, J. L. VanLente, and C. R. Phipps. 2002. Efficacy of systemic ceftiofur as a therapy for severe clinical mastitis in dairy cattle. J. Dairy Sci. 85:2571-2575.

Granier, S. A., V. Leflon-Guibout, F. W. Goldstein, and M. H. Nicolas-Chanoine. 2003. Enterobacterial repetitive intergenic consensus 1R PCR assay for detection of Raoultella sp. isolates among strains identified as Klebsiella oxytoca in the clinical laboratory. J. Clin. Microbiol. 41:1740-1742.

Gröhn, Y. T., R. N. González, D. J. Wilson, J. A. Hertl, G. Bennett, H. Schulte, and Y. H. Schukken. 2005. Effect of pathogen-specific clinical mastitis on herd life in two New York State dairy herds. Prev. Vet. Med. 71:105-125.

Gröhn, Y. T., D. J. Wilson, R. N. González, J. A. Hertl, H. Schulte, G. Bennett, and Y. H. Schukken. 2004. Effect of pathogen-specific clinical mastitis on milk yield in dairy cows. J. Dairy Sci. $87: 3358-3374$

Iniguez, A. L., Y. Dong, and E. W. Triplett. 2004. Nitrogen fixation in wheat provided by Klebsiella pneumoniae 342. Mol. Plant Microbe Interact. 17:1078-1085.

Kristula, M. A., Z. Dou, J. D. Toth, B. I. Smith, N. Harvey, and M. Sabo. 2008. Evaluation of free-stall mattress bedding treatments to reduce mastitis bacterial growth. J. Dairy Sci. 91:1885-1892.
Munoz, M. A., C. Ahlström, B. J. Rauch, and R. N. Zadoks. 2006. Fecal shedding of Klebsiella pneumoniae by dairy cows. J. Dairy Sci. 89:3425-3430.

Munoz, M. A., G. J. Bennett, C. Ahlström, H. M. Griffiths, Y. H. Schukken, and R. N. Zadoks. 2008. Cleanliness scores as indicator of Klebsiella exposure in dairy cows. J. Dairy Sci. 91:3908-3916.

Munoz, M. A., F. L. Welcome, Y. H. Schukken, and R. N. Zadoks. 2007. Molecular epidemiology of two Klebsiella pneumoniae mastitis outbreaks on a dairy farm in New York State. J. Clin. Microbiol. 45:3964-3971

Munoz, M. A., and R. N. Zadoks. 2007. Short communication: Patterns of fecal shedding of Klebsiella by dairy cows. J. Dairy Sci. 90:1220-1224.

National Mastitis Council. 1999. Laboratory Handbook on Bovine Mastitis. National Mastitis Council, Madison, WI

Newman, L. E., and J. J. Kowalski. 1973. Fresh sawdust bedding-A possible source of Klebsiella organisms. Am. J. Vet. Res. 34:979980

O'May, G. A., N. Reynolds, and G. T. Macfarlane. 2005. Effect of $\mathrm{pH}$ on an in vitro model of gastric microbiota in enteral nutrition patients. Appl. Environ. Microbiol. 71:4777-4783.

Olde Riekerink, R. G., H. W. Barkema, D. F. Kelton, and D. T. Scholl. 2008. Incidence rate of clinical mastitis on Canadian dairy farms. J. Dairy Sci. 91:1366-1377.

Roberson, J. R., L. D. Warninck, and G. Moore. 2004. Mild to moderate clinical mastitis: Efficacy of intramammary amoxicillin, frequent milk-out, a combined intramammary amoxicillin and frequent milk-out treatment versus no treatment. J. Dairy Sci. $87: 583-592$.

Russell, J. B., F. Diez-Gonzalez, and G. N. Jarvis. 2000. Potential effect of cattle diets on the transmission of pathogenic Escherichia coli to humans. Microbes Infect. 2:45-53.

Wenz, J. R., G. M. Barrington, F. B. Garry, K. D. McSweeney, R. P. Dinsmore, G. Goodell, and R. J. Callan. 2001. Bacteremia associated with naturally occurring acute coliform mastitis in dairy cows. J. Am. Vet. Med. Assoc. 219:976-981.

Wilson, D. J., Y. T. Gröhn, G. J. Bennett, R. N. González, Y. H. Schukken, and J. Spatz. 2007. Comparison of J5 vaccinates and controls for incidence, etiologic agent, clinical severity, and survival in the herd following naturally occurring cases of clinical mastitis. J. Dairy Sci. 90:4282-4288. 